\section{A182 INTERSTITIAL LUNG DISEASE IN PATIENTS WITH SCLERODERMA: TREATMENT WITH RITUXIMAB}

A Muñoz, ${ }^{1}$ R Martínez, 1 M León, ${ }^{1}$ F Gallo, ${ }^{1}$ M L Velloso, ${ }^{1}$ S Rodríguez, ${ }^{1}$ L Mayordomo, ${ }^{1}$ E Rejón, J L Marenco ${ }^{1}$ Rheumatology Unit, Valme University Hospital, Seville, Spain

10.1136/ard.2010.149013.25

Introduction Interstitial lung disease (ILD) is a severe complication of systemic sclerosis, more common among patients with diffuse scleroderma. Cyclophosphamide (CYC) is the treatment normally used in those patients, with irregular response. The authors have successfully treated three patients refractory to CYC with rituximab (RTX).

Patients and methods Patient 1: A 43-year-old woman developed progressive cough and dyspnea, with radiological changes of ILD. She began treatment with oral glucocorticoids and monthly boluses of CYC (1 g intravenously). Patient 2: A 53 -year-old woman with a diagnosis of diffuse scleroderma 3 years earlier, started with dyspnea during low strain at the beginning. ILD was diagnosed with characteristic ground glass pattern on thoracic CT. She was given six boluses of CYC and oral glucocorticoids. Patient 3: A 48-year-old women, with skin lesions suggestive of dermatomyositis (DM). In 2007 she was diagnosed of amyopathic DM with scleroderma overlap. Three 3 months after the diagnosis, she began with cough and dry expectoration, together with dyspnea. ILD was diagnosed with characteristic ground glass pattern on thoracic CT. She was initially treated monthly with CYC for 7 months. All patients, the authors started a treatment with RTX $(2 \times 1 \mathrm{~g})$ plus CYC.

Results Respiratory function tests showed improvements (see table 1).

Conclusions Overexpression of B lymphocytes in Scleroderma pathogenesis, and the bad evolution of ILD in those patients aimed them to try RTX (anti-CD-20) after CYC failure. The authors have observed clinical improvement with respiratory test (table 1) without progression in lung CT changes. Further studies would be necessary to establish this therapeutic approach.

Table 1

\begin{tabular}{|c|c|c|c|c|c|c|}
\hline & \multicolumn{2}{|l|}{ Patient 1} & \multicolumn{2}{|l|}{ Patient 2} & \multicolumn{2}{|l|}{ Patient 3} \\
\hline & Pre-RTX & Post-RTX & Pre-RTX & Post-RTX & Pre-RTX & Post-RTX \\
\hline Vital cap (I) & $1.94(65.2 \%)$ & $2.02(68.5 \%)$ & $1.08(44.8 \%)$ & $1.15(48.3 \%)$ & $1.43(53.4 \%)$ & $1.51(56.7 \%)$ \\
\hline FEV1/VC & 90.35 & 89.69 & 99.91 & 94.86 & 78.38 & 78.15 \\
\hline TLCOc/VA (mol/min/kpa) & $1.20(68 \%)$ & $1.03(59.2 \%)$ & $0.93(54.8 \%)$ & $1.02(60.6 \%)$ & $1.20(68.0 \%)$ & $1.29(73.8 \%)$ \\
\hline PC02 (mm Hg) & 44.1 & 42.8 & 32.6 & 40.2 & 33 & 35.1 \\
\hline
\end{tabular}

RTX, rituximab. 